\title{
Supporting Information: Extracting multi-way chromatin contacts from Hi-C data
}

Lei Liu and Bokai Zhang

Key Laboratory of Optical Field Manipulation of Zhejiang Province, Department of Physics, Zhejiang Sci-Tech University, Hangzhou, China

Changbong Hyeon

Korea Institute for Advanced Study, Seoul 02455, South Korea

\section{S1 APPENDIX}

Derivation of $n$-body contact probability based on the cross-linking probability modeled with the Heaviside step function.

\section{Pairwise contact probability}

Along with Eq. 9, the pairwise contact probability assuming $F_{1}(r)$ can be determined by [1]

$$
\begin{aligned}
p_{i j}^{(1)} & =\int_{0}^{\infty} P\left(r_{i j}\right) \Theta\left(r_{c}-r_{i j}\right) d r_{i j}=\int_{0}^{r_{c}} P\left(r_{i j}\right) d r_{i j} \\
& =\operatorname{erf}\left(\gamma_{i j}^{1 / 2} r_{c}\right)-2 r_{c} \sqrt{\frac{\gamma_{i j}}{\pi}} e^{-\gamma_{i j} r_{c}^{2}}
\end{aligned}
$$

with $\operatorname{erf}(x)=\frac{2}{\sqrt{\pi}} \int_{0}^{x} d t e^{-t^{2}}$.

\section{Three-body contact probability}

The simultaneous contact probability among three sites $i, j$, and $k$ assuming $F_{1}, p_{i j k}^{(1)}$, can be defined as

$$
\begin{aligned}
p_{i j k}^{(1)} & \equiv p\left(\left(r_{i j} \leq r_{c}\right) \wedge\left(r_{i k} \leq r_{c}\right) \wedge\left(r_{j k} \leq r_{c}\right)\right) \\
& =\int_{0}^{r_{c}} p\left(\left(r_{i k} \leq r_{c}\right) \wedge\left(r_{j k} \leq r_{c}\right) \mid r_{i j}\right) P\left(r_{i j}\right) d r_{i j}
\end{aligned}
$$

where $p\left(\left(r_{i k} \leq r_{c}\right) \wedge\left(r_{j k} \leq r_{c}\right) \mid r_{i j}\right)\left(\equiv p_{i k, j k \mid r_{i j}}^{(1)}\right)$ stands for the probability of the $k$-th monomer being simultaneously in contact with other two monomers conditioned with the distance $r_{i j}$, which we evaluated using cylindrical coordinates $(\rho, \phi, z)$. Under the conditions of $\vec{r}_{i}=\left(0,0, r_{i j} / 2\right)$ and $\vec{r}_{j}=\left(0,0,-r_{i j} / 2\right)$, the position of $k$-th monomer is still described in terms of the Gaussian distribution

$$
P\left(\vec{r}_{k}\right)=C e^{-\gamma_{i j, k}\left(z_{k}-z_{i j, k}^{*}\right)^{2}} e^{-\gamma_{i j, k} \rho_{k}^{2}},
$$

with the variance of the distribution $\gamma_{i j, k}=$ $\operatorname{det}\left(\mathbf{K}_{i j}\right) / \operatorname{det}\left(\mathbf{K}_{i j k}\right)$, and a normalization constant $C=$ $\left(\pi / \gamma_{i j, k}\right)^{-3 / 2} \cdot P\left(\vec{r}_{k}\right)$ is shifted along $z$-axis by

$$
z_{i j, k}^{*}=\frac{r_{i j}}{2}\left\{\left(\mathbf{K}_{i j}\right)^{-1} \cdot\left(\mathbf{k}_{\backslash\{i, j\}}^{j}-\mathbf{k}_{\backslash\{i, j\}}^{i}\right)\right\}_{k},
$$

where $\mathbf{k}_{\backslash\{i, j\}}^{m}$ is the $m$-th column of the matrix $\mathbf{K}$ after removing the $i$-th and the $j$-th rows. For $r_{i j} \leq r_{c}$, the conditional probability $q_{i k, j k \mid r_{i j}}$ equals to an integral over the intersection formed between two spheres of a radius $r_{c}$

$$
\begin{aligned}
p_{i k, j k \mid r_{i j}}^{(1)}= & \int_{0}^{2 \pi} d \phi \int_{-D}^{D} d z \int_{0}^{R(z)} d \rho P\left(\vec{r}_{k}\right) \rho \\
= & C \int_{0}^{2 \pi} d \phi \int_{-D}^{D} d z e^{-\gamma_{i j, k}\left(z-z_{i j, k}^{*}\right)^{2}} \int_{0}^{R(z)} d \rho e^{-\gamma_{i j, k} \rho^{2}} \rho \\
= & -\left.\sqrt{\frac{\gamma_{i j, k}}{\pi}} \int_{-D}^{D} d z e^{-\gamma_{i j, k}\left(z-z_{i j, k}^{*}\right)^{2}} e^{-\gamma_{i j, k} \rho^{2}}\right|_{\rho=0} ^{\rho=R(z)} \\
= & \sqrt{\frac{\gamma_{i j, k}}{\pi}}\left[\int_{-D}^{D} d z e^{-\gamma_{i j, k}\left(z-z_{i j, k}^{*}\right)^{2}}\right. \\
& -e^{-\gamma_{i j, k} r_{c}^{2}} \int_{-D}^{0} d z e^{-\gamma_{i j, k}\left(z-z_{i j, k}^{*}\right)^{2}} e^{\gamma_{i j, k}\left(\frac{r_{i j}}{2}-z\right)^{2}} \\
& \left.-e^{-\gamma_{i j, k} r_{c}^{2}} \int_{0}^{D} d z e^{-\gamma_{i j, k}\left(z-z_{i j, k}^{*}\right)^{2}} e^{\gamma_{i j, k}\left(\frac{r_{i j}}{2}+z\right)^{2}}\right] \\
= & \sqrt{\frac{\gamma_{i j, k}}{\pi}}\left[I_{0}+e^{-\gamma_{i j, k} r_{c}^{2}} I_{-}+e^{-\gamma_{i j, k} r_{c}^{2}} I_{+}\right],
\end{aligned}
$$

where $R(z)=\left(r_{c}^{2}-\left(\frac{r_{i j}}{2}+|z|\right)^{2}\right)^{1 / 2}$ has been inserted in the third row. Considering that $D=r_{c}-r_{i j} / 2(>0)$, it is straightforward to calculate the integrals in the second to the last row of Eq. S5. One gets

$$
I_{0}=\left\{\begin{array}{ll}
\frac{1}{2} \sqrt{\frac{\pi}{\gamma_{i j, k}}}\left[\operatorname{erf}\left(\left|z_{-}\right|\right)+\operatorname{erf}\left(\left|z_{+}\right|\right)\right], & z_{-} z_{+}<0 \\
\frac{1}{2} \sqrt{\frac{\pi}{\gamma_{i j, k}}}\left|\operatorname{erf}\left(\left|z_{-}\right|\right)-\operatorname{erf}\left(\left|z_{+}\right|\right)\right|, & z_{-} z_{+} \geq 0
\end{array},\right.
$$

with $z_{\mp}=\left(\mp D-z_{i j, k}^{*}\right) \gamma_{i j, k}^{1 / 2}$, and

$$
I_{\mp}=\left\{\begin{array}{ll}
-D, & r_{\mp}=0 \\
\frac{e^{\left(\gamma_{i j, k} r_{+} r_{-}\right) / 4}}{\gamma_{i j, k} r_{\mp}}\left(1-e^{\gamma_{i j, k} D r_{\mp}}\right), & r_{\mp} \neq 0
\end{array},\right.
$$

where $r_{\mp}=r_{i j} \pm 2 z_{i j, k}^{*}$. The triplet contact probability in Eq.S2 can be computed numerically as a one-dimensional finite integral of a function of variable $r_{i j}$, together with Eqs. 9, S5, S7 


\section{Conditional pairwise contact probability}

Similarly to the discussions in the Subsection: Comparison with SPRITE in the main text, the first conditional pairwise contact probability, $p\left(r_{j k} \leq r_{c} \mid r_{i j} \leq r_{c}\right)$, can be calculated based on the relation that $p\left(r_{j k} \leq r_{c} \mid\right.$ $\left.r_{i j} \leq r_{c}\right)=p_{j k \mid r_{i j} \leq r_{c}}^{(1)}=p_{i j, j k}^{(1)} / p_{i j}^{(1)}$, where

$$
\begin{aligned}
p_{i j, j k}^{(1)} & =p\left(\left(r_{j k} \leq r_{c}\right) \wedge\left(r_{i j} \leq r_{c}\right)\right) \\
& =\int_{0}^{r_{c}} P\left(r_{i j}\right) p\left(r_{j k} \leq r_{c} \mid r_{i j}\right) d r_{i j} .
\end{aligned}
$$

By setting $\vec{r}_{i}=\left(0,0, r_{i j}\right)$ and $\vec{r}_{j}=(0,0,0)$, we calculated $p\left(r_{j k} \leq r_{c} \mid r_{i j}\right)\left(\equiv p_{j k \mid r_{i j}}^{(1)}\right)$, via a similar procedure of obtaining $p_{i k, j k \mid r_{i j}}^{(1)}$ (Eq S5. The distribution of the $k$-th monomer, $P\left(\vec{r}_{k}\right)$, has the same form as Eq. [S3, with the center being determined at $z_{i j, k}^{*}=$ $r_{i j}\left\{\left(\mathbf{K}_{i j}\right)^{-1} \cdot\left(-\mathbf{k}_{\backslash\{i, j\}}^{i}\right)\right\}_{k} . \quad p_{j k \mid r_{i j}}^{(1)}$ is evaluated by integrating over a sphere centered at the origin with a radius $r_{c}$,

$$
\begin{aligned}
p_{j k \mid r_{i j}}^{(1)} & =\int_{0}^{2 \pi} d \phi \int_{-r_{c}}^{r_{c}} d z \int_{0}^{R(z)} d \rho P\left(\vec{r}_{k}\right) \rho \\
& =\sqrt{\frac{\gamma_{i j, k}}{\pi}}\left[\int_{-r_{c}}^{r_{c}} d z e^{-\gamma_{i j, k}\left(z-z_{i j, k}^{*}\right)^{2}}\right. \\
& \left.-\int_{-r_{c}}^{r_{c}} d z e^{-\gamma_{i j, k}\left(z-z_{i j, k}^{*}\right)^{2}} e^{-\gamma_{i j, k}\left(r_{c}^{2}-z^{2}\right)}\right] \\
& =\sqrt{\frac{\gamma_{i j, k}}{\pi}}\left[I_{0}-I_{1}\right],
\end{aligned}
$$

where $R(z)=\left(r_{c}^{2}-z^{2}\right)^{1 / 2}, I_{0}$ is given in Eq. S6 with $z_{\mp}=\left(\mp r_{c}-z_{i j, k}^{*}\right) \gamma_{i j, k}^{1 / 2}$, and

$$
I_{1}=\left\{\begin{array}{ll}
2 r_{c} e^{-\gamma_{i j, k}\left(z_{i j, k}^{* 2}+r_{c}^{2}\right)}, & z_{i j, k}^{*}=0 \\
\frac{e^{-\gamma_{i j, k}\left(z_{i j, k}^{* 2}+r_{c}^{2}\right)}}{\gamma_{i j, k} z_{i j, k}^{*}} \sinh \left(2 \gamma_{i j, k} z_{i j, k}^{*} r_{c}\right), & z_{i j, k}^{*} \neq 0
\end{array} .\right.
$$

After computing $p_{i j, j k}^{(1)}$ with Eq. S8 $\mathrm{S} 9$, the second conditional pairwise contact probability can be determined by

$$
p_{j k \mid r_{i j}>r_{c}}^{(1)}=\frac{p_{j k}^{(1)}-p_{i j, j k}^{(1)}}{1-p_{i j}^{(1)}}
$$

where the marginal contact probabilities $p_{i j}^{(1)}$ and $p_{j k}^{(1)}$ are given by Eq. S1 in this case.

Note that neither $p_{j k \mid r_{i j} \leq r_{c}}^{(1)}$ nor $p_{i j, j k}^{(1)}$ equals to $p_{i j k}^{(1)}$ if $r_{c}>0$. Although both loci are concurrently in contact with the $j$-th site, the contact between the $i$-th and $k$ th loci is not guaranteed. To be precise, what Bintu et al. have quantified 2] are not higher-order chromatin contacts. 
TABLE A. Genomic regions simulated in this work.

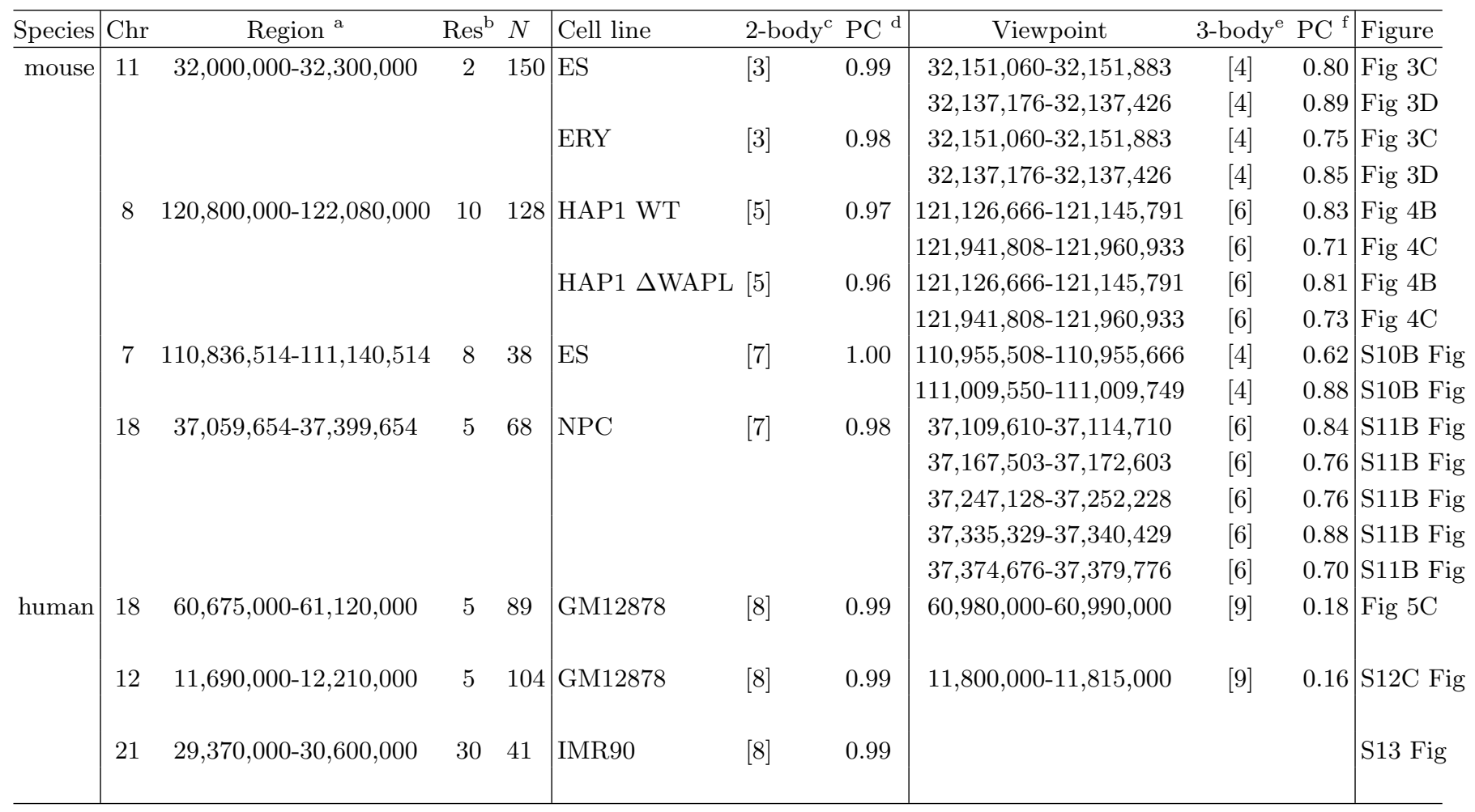

a The reference genome assemblies of mouse and human are mm9 and hg19, respectively.

b The model resolutions in units of $\mathrm{kb}$.

c References of the experimental pairwise contacts datasets.

${ }^{\mathrm{d}}$ Pearson correlation coefficient of two-body contact probabilities between Hi-C/Capture-C and HLM.

e References of the experimental triplet contacts datasets.

${ }^{\mathrm{f}}$ Pearson correlation coefficient of three-body contact probabilities between 3-body experiments and HLM.

TABLE B. Pearson correlation (PC), stratum-adjusted correlation (SCC [10]) and distance-corrected Pearson correlation (DCPC [1] ) of the contact probabilities predicted by SBS [12] and HLM compared with Capture-C [3] and Tri-C [4] experiments ("NA" stands for not available).

\begin{tabular}{|c|c|c|c|c|c|c|c|}
\hline & \multicolumn{2}{|c|}{ Pairwise contacts } & \multicolumn{2}{|c|}{ Triplet contacts at $\mathrm{R} 2$} & \multicolumn{2}{|c|}{ Triplet contacts at HS-39 } \\
\hline & & (SBS, Cap-C) & (HLM, Cap-C) & (SBS, Tri-C) & (HLM, Tri-C) & (SBS, Tri-C) & (HLM, Tri-C) \\
\hline $\mathrm{ES}$ & $\mathrm{PC}$ & 0.96 & 0.99 & 0.80 & 0.80 & 0.84 & 0.89 \\
\hline & $\mathrm{SCC}$ & 0.75 & 0.88 & 0.66 & 0.72 & 0.77 & 0.82 \\
\hline & $\mathrm{DCPC}$ & 0.87 & 0.91 & NA & 0.68 & NA & 0.65 \\
\hline ERY & $\mathrm{PC}$ & 0.96 & 0.98 & 0.80 & 0.75 & 0.77 & 0.85 \\
\hline & $\mathrm{SCC}$ & 0.92 & 0.93 & 0.48 & 0.66 & 0.79 & 0.80 \\
\hline & DCPC & 0.91 & 0.97 & NA & 0.70 & NA & 0.72 \\
\hline
\end{tabular}


TABLE C. Stratum adjusted correlation (SCC) and PC coefficients between Hi-C and HLM model, which was trained with different cross-linking probabilities, cost functions, and optimizers (see also S12 Fig). Each coefficient value is the averaged result of five independent trainings.

\begin{tabular}{cr|rrrr}
\hline & & RS & GD & RMS & ADAM \\
\hline SCC $F_{0}$ & $L_{0}$ & $-1.872 \mathrm{e}-02$ & $5.684 \mathrm{e}-02$ & $7.326 \mathrm{e}-01$ & $7.250 \mathrm{e}-01$ \\
& $L_{1}$ & $7.700 \mathrm{e}-02$ & $7.400 \mathrm{e}-01$ & $7.936 \mathrm{e}-01$ & $\mathbf{8 . 4 1 8 e - 0 1}$ \\
& $L_{2}$ & $7.191 \mathrm{e}-01$ & $5.811 \mathrm{e}-01$ & $8.159 \mathrm{e}-01$ & $\mathbf{8 . 5 3 7 e - 0 1}$ \\
& $L_{3}$ & $-1.872 \mathrm{e}-02$ & $9.641 \mathrm{e}-02$ & $7.960 \mathrm{e}-01$ & $8.390 \mathrm{e}-01$ \\
\hline PC $F_{0}$ & $L_{0}$ & $8.194 \mathrm{e}-01$ & $9.378 \mathrm{e}-01$ & $9.829 \mathrm{e}-01$ & $9.835 \mathrm{e}-01$ \\
& $L_{1}$ & $8.731 \mathrm{e}-01$ & $9.818 \mathrm{e}-01$ & $9.884 \mathrm{e}-01$ & $\mathbf{9 . 9 1 4 e - 0 1}$ \\
& $L_{2}$ & $9.836 \mathrm{e}-01$ & $9.753 \mathrm{e}-01$ & $9.901 \mathrm{e}-01$ & $\mathbf{9 . 9 2 4 e - 0 1}$ \\
& $L_{3}$ & $8.1934-01$ & $9.025 \mathrm{e}-01$ & $9.883 \mathrm{e}-01$ & $9.913 \mathrm{e}-01$ \\
\hline SCC $F_{1}$ & $L_{0}$ & $6.318 \mathrm{e}-02$ & $6.798 \mathrm{e}-02$ & $7.661 \mathrm{e}-01$ & $8.073 \mathrm{e}-01$ \\
& $L_{1}$ & $3.944 \mathrm{e}-01$ & $7.488 \mathrm{e}-01$ & $7.933 \mathrm{e}-01$ & $8.441 \mathrm{e}-01$ \\
& $L_{2}$ & $7.161 \mathrm{e}-01$ & $5.809 \mathrm{e}-01$ & $8.155 \mathrm{e}-01$ & $\mathbf{8 . 5 4 3 e - 0 1}$ \\
& $L_{3}$ & $8.207 \mathrm{e}-02$ & $1.040 \mathrm{e}-01$ & $7.956 \mathrm{e}-01$ & $\mathbf{8 . 4 5 4 e - 0 1}$ \\
\hline PC $F_{1}$ & $L_{0}$ & $8.561 \mathrm{e}-01$ & $9.339 \mathrm{e}-01$ & $9.843 \mathrm{e}-01$ & $9.900 \mathrm{e}-01$ \\
& $L_{1}$ & $9.423 \mathrm{e}-01$ & $9.825 \mathrm{e}-01$ & $9.884 \mathrm{e}-01$ & $9.917 \mathrm{e}-01$ \\
& $L_{2}$ & $9.834 \mathrm{e}-01$ & $9.753 \mathrm{e}-01$ & $9.901 \mathrm{e}-01$ & $\mathbf{9 . 9 2 4 e - 0 1}$ \\
& $L_{3}$ & $8.627 \mathrm{e}-01$ & $9.003 \mathrm{e}-01$ & $9.885 \mathrm{e}-01$ & $\mathbf{9 . 9 1 8 e - 0 1}$ \\
\hline
\end{tabular}


[1] Liu L, Kim MH, Hyeon C. Heterogeneous loop model to infer 3D chromosome structures from Hi-C. Biophys J. 2019;117(3):613-625. doi:10.1016/j.bpj.2019.06.032.

[2] Bintu B, Mateo LJ, Su JH, Sinnott-Armstrong NA, Parker M, Kinrot S, et al. Super-resolution chromatin tracing reveals domains and cooperative interactions in single cells. Science. 2018;362(6413):eaau1783. doi:10.1126/science.aau1783.

[3] Oudelaar AM, Beagrie RA, Gosden M, de Ornellas S, Georgiades E, Kerry J, et al. Dynamics of the 4D genome during in vivo lineage specification and differentiation. Nat Comm. 2020;11(1):2722. doi:10.1038/s41467-02016598-7.

[4] Oudelaar AM, Davies JOJ, Hanssen LLP, Telenius JM, Schwessinger R, Liu Y, et al. Single-allele chromatin interactions identify regulatory hubs in dynamic compartmentalized domains. Nat Genet. 2018;50:1744-1751. doi:10.1038/s41588-018-0253-2.

[5] Haarhuis JHI, van der Weide RH, Blomen A Vincent, Yáñez-Cuna JO, Amendola M, van Ruiten MS, et al. The Cohesin Release Factor WAPL Restricts Chromatin Loop Extension. Cell. 2017;169(4):693-707. doi:10.1016/j.cell.2017.04.013.

[6] Allahyar A, Vermeulen C, Bouwman BAM, Krijger PHL, Verstegen MJAM, Geeven G, et al. Enhancer hubs and loop collisions identified from single-allele topologies. Nat Genet. 2018;50(8):1151-1160. doi:10.1038/s41588-0180161-5.

[7] Bonev B, Cohen NM, Szabo Q, Fritsch L, Papadopoulos GL, Lubling Y, et al. Multiscale 3D Genome Rewiring during Mouse Neural Development. Cell. 2017;171(3):557 - 572.e24. doi:https://doi.org/10.1016/j.cell.2017.09.043.

[8] Rao SSP, Huntley MH, Durand NC, Stamenova EK, Bochkov ID, Robinson JT, et al. A 3D Map of the Human Genome at Kilobase Resolution Reveals Principles of Chromatin Looping. Cell. 2014;159(7):1665-1680. doi:10.1016/j.cell.2014.11.021.

[9] Quinodoz SA, Ollikainen N, Tabak B, Palla A, Schmidt JM, Detmar E, et al. Higher-Order Interchromosomal Hubs Shape 3D Genome Organization in the Nucleus. Cell. 2018;174(3):744 - 757.e24. doi:https://doi.org/10.1016/j.cell.2018.05.024.

[10] Yang T, Zhang F, Yardimci GG, Song F, Hardison RC, Noble WS, et al. HiCRep: assessing the reproducibility of Hi-C data using a stratum-adjusted correlation coefficient. Genome Res. 2017;27:1939-1949. doi:10.1101/gr.220640.117.

[11] Bianco S, Lupiáñez DG, Chiariello AM, Annunziatella C, Kraft K, Schöpflin R, et al. Polymer physics predicts the effects of structural variants on chromatin architecture. Nat Genetics. 2018;50:662-667.

[12] Chiariello AM, Bianco S, Oudelaar AM, Esposito A, Annunziatella C, Fiorillo L, et al. A Dynamic Folded Hairpin Conformation Is Associated with alpha-Globin Activation in Erythroid Cells. Cell Rep. 2020;30(7):2125-2135. doi:10.1016/j.celrep.2020.01.044.
[13] Liu L, Kim MH, Hyeon C. Heterogeneous loop model to infer 3D chromosome structures from Hi-C. Biophys J. 2019;117(3):613-625. doi:10.1016/j.bpj.2019.06.032.

[14] Bintu B, Mateo LJ, Su JH, Sinnott-Armstrong NA, Parker M, Kinrot S, et al. Super-resolution chromatin tracing reveals domains and cooperative interactions in single cells. Science. 2018;362(6413):eaau1783. doi:10.1126/science.aau1783.

[15] Oudelaar AM, Beagrie RA, Gosden M, de Ornellas S, Georgiades E, Kerry J, et al. Dynamics of the 4D genome during in vivo lineage specification and differentiation. Nat Comm. 2020;11(1):2722. doi:10.1038/s41467-02016598-7.

[16] Oudelaar AM, Davies JOJ, Hanssen LLP, Telenius JM, Schwessinger R, Liu Y, et al. Single-allele chromatin interactions identify regulatory hubs in dynamic compartmentalized domains. Nat Genet. 2018;50:1744-1751. doi:10.1038/s41588-018-0253-2.

[17] Haarhuis JHI, van der Weide RH, Blomen A Vincent, Yáñez-Cuna JO, Amendola M, van Ruiten MS, et al. The Cohesin Release Factor WAPL Restricts Chromatin Loop Extension. Cell. 2017;169(4):693-707. doi:10.1016/j.cell.2017.04.013.

[18] Allahyar A, Vermeulen C, Bouwman BAM, Krijger PHL, Verstegen MJAM, Geeven G, et al. Enhancer hubs and loop collisions identified from single-allele topologies. Nat Genet. 2018;50(8):1151-1160. doi:10.1038/s41588-0180161-5.

[19] Bonev B, Cohen NM, Szabo Q, Fritsch L, Papadopoulos GL, Lubling Y, et al. Multiscale 3D Genome Rewiring during Mouse Neural Development. Cell. 2017;171(3):557 - 572.e24. doi:https://doi.org/10.1016/j.cell.2017.09.043.

[20] Rao SSP, Huntley MH, Durand NC, Stamenova EK, Bochkov ID, Robinson JT, et al. A 3D Map of the Human Genome at Kilobase Resolution Reveals Principles of Chromatin Looping. Cell. 2014;159(7):1665-1680. doi:10.1016/j.cell.2014.11.021.

[21] Quinodoz SA, Ollikainen N, Tabak B, Palla A, Schmidt JM, Detmar E, et al. Higher-Order Interchromosomal Hubs Shape 3D Genome Organization in the Nucleus. Cell. 2018;174(3):744 - 757.e24. doi:https://doi.org/10.1016/j.cell.2018.05.024.

[22] Yang T, Zhang F, Yardimci GG, Song F, Hardison RC, Noble WS, et al. HiCRep: assessing the reproducibility of Hi-C data using a stratum-adjusted correlation coefficient. Genome Res. 2017;27:1939-1949. doi:10.1101/gr.220640.117.

[23] Bianco S, Lupiáñez DG, Chiariello AM, Annunziatella C, Kraft K, Schöpflin R, et al. Polymer physics predicts the effects of structural variants on chromatin architecture. Nat Genetics. 2018;50:662-667.

[24] Chiariello AM, Bianco S, Oudelaar AM, Esposito A, Annunziatella C, Fiorillo L, et al. A Dynamic Folded Hairpin Conformation Is Associated with alpha-Globin Activation in Erythroid Cells. Cell Rep. 2020;30(7):2125-2135. doi:10.1016/j.celrep.2020.01.044. 